\title{
Producción de prostaciclina en arteria radial de diabéticos: Comparación con la vena safena y arteria mamaria interna y su relevancia en el pronóstico de estos bypass coronarios
}

\author{
Mauricio G uivernau리 Miguel Berr². \\ Prostacyclin production in internal \\ mammary and radial arteries and \\ saphenous veins of diabetic and non \\ diabetic subjects
}

Background: Myocardial revascularization surgery has used several vessels as coronary grafts including internal mammary and radial arteries which have a better prognosis than saphenous vein. Their long-term patency has been associated with the release of endothelium vasodilator and anti-aggregating products such as prostacyclin. Diabetes induces endothelial dysfunction and a high number of diabetics require revascularization. Aim: To assess the capacity to synthesize prostacyclin of different vessels from diabetics. Material and methods: Internal mammary and radial arteries and saphenous veins obtained from 10 diabetic and 10 non diabetic patients subjected to coronary artery bypass surgery were studied. The capacity to synthesize prostacyclin was assessed in these vessels measuring its hydrolysis product, the 6-keto-PGF1 $\alpha$ by radioimmunoassay. Results: Internal mammary arteries and saphenous veins from diabetics synthesized a lower amount of prostacyclin than those from non-diabetics. The radial artery produced similar amounts of prostacyclin in both groups. This response was associated with an increase of the conversion of the precursor arachidonic acid to prostacyclin. The saturating concentrations of this acid required to achieve the maximal stimulation were higher in the radial artery $(20 \mu \mathrm{M})$ than in the internal mammary artery and saphenous vein $(10 \mu \mathrm{M})$, suggesting that the enzymatic activity of the radial artery was not affected by diabetes. Conclusions: The radial artery appears as the best replacement vessel for coronary surgery in diabetics. Its favorable biochemical profile and potential lower long-term occlusion rate may be relevant for a better prognosis of myocardial revascularization in these patients (Rev Méd Chile 2008; 136: 823-30).

(Key words: Diabetes complications; Coronary artery disease; Epoprostenol; Mammary arteries; Radial artery)

Recibido el 12 de septiembre, 2007. Aceptado el 10 de marzo, 2008.

${ }^{1}$ Programa de Farmacología Molecular y Clínica, Instituto de Ciencias Biomédicas, Facultad de Medicina, Universidad de Chile, Santiago de Chile. ${ }^{2}$ Hospital DIPRECA, Santiago de Chile.

Correspondencia a: Dr. Mauricio Guivernau. Programa de Farmacología Molecular y Clínica, ICBM, Facultad de Medicina, Universidad de Chile. Independencia 1027, Casilla 70.000, Santiago 7, Chile. Fax: (56-2) 7372783. Fono: (56-2) 9786056. E mail: mguivern@med.uchile.cl 
L a búsqueda de bypass coronarios óptimos para la revascularización del miocardio ha representado un desafío permanente en cirugía cardiovascular. El primer vaso utilizado fue la vena safena; sin embargo, estudios a largo plazo han evidenciado un mal pronóstico debido a su elevada tasa de oclusión ${ }^{1}$. Posteriormente, se han utilizado conductos arteriales, tales como las arterias mamaria interna, epigástrica inferior y radial, que han mostrado un mejor pronóstico ${ }^{2}$. Diversos estudios muestran que estas arterias sintetizan cantidades mayores de prostaciclina que la vena safena ${ }^{3}$. Trabajos nuestros evidencian resultados similares ${ }^{4}$, destacando que la arteria radial es capaz de producirla en cantidades similares a las otras dos arterias señaladas ${ }^{5}$. Últimamente, ha renacido el interés por la arteria radial ${ }^{6}$, particularmente en revascularización lograda mediante el uso simultáneo de dos o más conductos arteriales ${ }^{7}$.

El éxito de la cirugía coronaria depende de la permeabilidad a largo plazo de estos conductos; los mecanismos bioquímicos y moleculares responsables de su inoclusión se han relacionado con la liberación en el endotelio vascular de mediadores vasoactivos, tales como la prostaciclina y tromboxano ${ }^{8}$, y el óxido nítrico ${ }^{9}$, que participan en los mecanismos responsables de la integridad y disfunción endotelial ${ }^{10}$. La prostaciclina es un potente modulador de las interacciones vasos-plaquetas, flujo sanguíneo tisular y efectos agregantes del tromboxano, además de inhibir la hiperplasia de la íntima y la activación de las plaquetas y acelerar la regeneración del endotelio ${ }^{11}$. Una producción disminuida de prostaciclina o aumentada de tromboxano expondrá la superficie de los vasos a una acumulación de plaquetas y liberación de factores endógenos que estimulan la proliferación de células musculares lisas en la pared vascular, iniciando la secuencia de eventos que culminan en aterosclerosis ${ }^{12}$. La prostaciclina es el principal eicosanoide sintetizado por el endotelio de los vasos. El primer paso en su biosíntesis consiste en la liberación de su precursor, el ácido araquidónico, desde los fosfolípidos de la membrana celular por acción de la enzima fosfolipasa A2, lugar donde este ácido graso esencial se almacena después de ser ingerido a través de la dieta. Una vez liberado, su conversión es iniciada por la enzima ciclooxigenasa (COX), que lo transforma primeramente en los endoperóxidos cíclicos (PGG2 y PGH2), los que a su vez son convertidos en fracción de segundos por enzimas tisulares locales en prostaciclina. La prostaciclina tiene una vida media muy corta y se degrada espontáneamente por hidrólisis no enzimática en su metabolito estable, el 6-ceto-PGF1 $\alpha$, que representa un índice de la prostaciclina formada y que se cuantifica por técnicas analíticas de radioinmunoensayo ${ }^{8}$.

Por otra parte, la asociación entre diabetes y aterosclemosis es bien conocida, existiendo un elevado porcentaje de diabéticos que requieren revascularización del miocardio ${ }^{13}$. Estos pacientes muestran una baja actividad de las enzimas responsables de la síntesis de prostaciclina ${ }^{14}$, acompañada de un aumento del tromboxano y de la agregación plaquetaria ${ }^{15}$, eventos claves en el desarmollo de la enfermedad tromboembólica. Algunos trabajos muestran una producción disminuida de prostaciclina en la arteria mamaria interna de diabéticos ${ }^{16}$, no habiéndose estudiado en este tipo de pacientes el comportamiento de otras arterias utilizadas como bypass coronarios. Interesantemente, se ha comunicado que el efecto protector vascular de la prostaciclina es más intenso en la arteria radial que en la mamaria intema ${ }^{17}$.

En este estudio se investigó la producción de prostaciclina en biopsias de segmentos distales que son rutinariamente descartados en este tipo de cirugía, de las arterias mamaria interna y radial y vena safena obtenidos de pacientes diabéticos sometidos a revascularización miocárdica, comparándola con la de no diabéticos. Nuestra hipótesis de trabajo planteó la posibilidad de que en diabéticos la arteria radial sea capaz de sintetizar mayores cantidades de prostaciclina que la mamaria interna; en atención a que este último factor juega un papel clave en el desarrollo de oclusión 0 reestenosis de los bypass coronarios, surge la posibilidad que la arteria radial represente un conducto complementario de mejor elección en diabéticos, lo cual se traduciría en un pronóstico a largo plazo más óptimo de la revascularización de miocardio.

\section{MATERIAL Y MÉTOdO}

Diseño del estudio. Las arterias mamaria interna y radial, así como la vena safena, se obtuvieron de 
10 pacientes diabéticos y 10 no diabéticos sometidos a cirugía coronaria electiva de revascularización del miocardio en el Hospital DIPRECA. La edad promedio de los pacientes fue de $58 \pm 7$ años. Pacientes con historia de falla cardiaca 0 renal crónica, enfermedad vascular periférica o sometidos a cirugía coronaria de urgencia fueron excluidos de este estudio. Los pacientes fueron informados previamente en detalle de la naturaleza del estudio de acuerdo a protocolos institucionales para el uso de tejidos humanos; los participantes dieron su consentimiento por escrito y el protocolo de estudio fue aprobado por el comité de ética de la institución. Es importante destacar que lo anterior se aplicó a pesar de que en todos los casos se utilizaron segmentos distales de estos vasos que son rutinariamente descartados en este tipo de cirugía.

Con el fin de conservar la funcionalidad del endotelio vascular, las biopsias obtenidas de los vasos antes mencionados se depositaron en forma inmediata en una solución de Krebs-Henseleit gasificada con $95 \%$ de $\mathrm{O} 2$ y $5 \%$ de $\mathrm{CO} 2$ y se congelaron y almacenaron a una temperatura de $-70^{\circ} \mathrm{C}$ durante $48 \mathrm{~h}$ después de las cuales se incubaron en la forma que se describe en el párrafo siguiente para estudiar su producción de prostaciclina.

Medición de prostaciclina. La producción vascular de prostaciclina se midió por técnicas analíticas de radioinmunoensayo (RIA) de su producto estable de hidrólisis espontánea no enzimática, el 6-cetoPGF1 $\alpha$, utilizando un kit comercial (Cayman Chemical Company, ANN Arbor, MI, USA, ํㅜ catálogo 416011), cuyo límite de detección fue de $3 \mathrm{pg} / \mathrm{ml}$. Las muestras de segmentos distales de arterias mamaria interna y radial y de vena safena fueron lavadas con solución salina, liberadas de tejido graso y conectivo y cortadas manualmente para obtener anillos de aproximadamente $2 \mathrm{~mm}$ de ancho. Tres a cinco de estos anillos, con un peso total aproximado de 2 a $3 \mathrm{mg}$, se incubaron en presencia y ausencia de suplementación del medio de incubación con ácido araquidónico, el precursor de la prostaciclina, en frascos sellados a $37^{\circ} \mathrm{C}$ en $3 \mathrm{ml}$ de una solución de Krebs-Henseleit de la siguiente composición $(\mathrm{mM} / \mathrm{l}): \mathrm{NaCl}, 118$; $\mathrm{KCl}, 5,4 ; \mathrm{MgSO}$ 4, 1,0; $\mathrm{CaCl}$ 2, 2,5; $\mathrm{NaHPO} 4,1,1$; NaHCO3, 25 y glucosa, 10. Esta solución fue burbujeada con una mezcla de dióxido de carbo- no $5 \%$ y oxígeno $95 \%$, y el pH final se ajustó a 7,4 . Después de diversos tiempos de incubación $(5,15$ y $30 \mathrm{~min}$ ), se extrajeron las fracciones líquidas del medio -libres de tejido vascular- conteniendo la prostaciclina sintetizada y se congelaron a $-70^{\circ} \mathrm{C}$ en espera de las mediciones, las cuales se realizaron procesando todas las muestras con un mismo kit de RIA. Las alícuotas fueron diluidas al 1:15 y 1:30 para lograr concentraciones de prostaciclina, que se ubicaran en la parte lineal de la curva del RIA y dentro del rango de detección del kit de radioinmunoensayo cuyo límite es de 14 picogramos. La reactividad cruzada del anticuerpo con otras prostaglandinas fue menor de $0,015 \%$. Los valores se expresaron en nanogramos de prostaciclina producidos por miligramo de tejido en los diferentes tiempos de incubación señalados (ng/ $\mathrm{mg} / \mathrm{min}$ ). La descripción detallada de esta metodología se puede encontrar en trabajos publicados anteriormente por los autores de este trabajo $^{18}$.

Estadística. Los resultados se expresaron como promedio \pm error estándar (E.E.). La significación estadística de las diferencias entre los vasos de los grupos diabéticos y no diabéticos se analizó por el test $\mathrm{t}$ de Student y cuando fue pertinente por ANOVA o por test post hoc Neuman-Keuls. Valores de $\mathrm{p}<0,05$ fueron considerados estadísticamente significativos.

\section{RESULTADOS}

Pacientes no diabéticos: En estos pacientes, los segmentos distales de las arterias mamaria interna (AMI) y radial (AR) sintetizaron en condiciones basales cantidades equivalentes de prostaciclina. En cambio, cuando se compararon con la vena safena (VS), ambas arterias produjeron cantidades significativamente mayores, como se puede observar en la Tabla 1. En efecto, cuando anillos de estos vasos se incubaron en solución de KrebsHenseleit, las diferencias entre ellos medida por radioinmunoensayo del 6-ceto-PGF1 $\alpha$, metabolito de hidrólisis no enzimática de la prostaciclina, se evidenciaron tanto a los 5, como a los 15 y $30 \mathrm{~min}$ de incubación de los tejidos; la producción de prostaciclina observada en todos los casos fue proporcional a la cantidad de tejido vascular utilizada. 


\section{Tabla 1. Producción basal de prostaciclina en la vena safena y arterias mamaria interna y radial obtenidas de pacientes no diabéticos}

\begin{tabular}{|lccc|}
\hline Vasos & \multicolumn{3}{c|}{ Tiempo de incubación (min) } \\
& 5 & 10 & 30 \\
\hline Vena safena & $0,4 \pm 0,1$ & $2,4 \pm 0,4$ & $3,7 \pm 1,2$ \\
Arteria mamaria interna & $1,6 \pm 0,3$ & $6,3 \pm 1,5$ & $12,3 \pm 2,6^{*} \mathrm{NS}$ \\
Arteria radial & $1,8 \pm 0,5$ & $6,0 \pm 1,3$ & $11,7 \pm 2,0^{*} \mathrm{NS}$ \\
\hline
\end{tabular}

La prostaciclina se midió por radioinmunoensayo de su producto estable 6-ceto-PGF1 $\alpha$. Los valores corresponden al promedio \pm error estándar (E.E.) y se expresan en $\mathrm{ng} / \mathrm{mg}$ de tejido/tiempo de incubación. ${ }^{*}$ p $<0,01$ para las comparaciones entre tejido venoso y arteriales; NS $=$ No significativo para las comparaciones entre las arterias mamaria interna y radial; $\mathrm{n}=10$ incubaciones para cada uno de los tres vasos estudiados y obtenidos de igual número de biopsias.

Esta mayor producción de prostaciclina en las dos arterias, comparada con la vena safena, se acentuó cuando el medio de incubación fue suplementado con $10 \mu \mathrm{M}$ de ácido araquidónico precursor de prostaciclina, una concentración capaz de producir la máxima estimulación de la síntesis de esta prostaglandina. Los resultados fueron: $\mathrm{VS}=6,2 \pm 1,9 ; \mathrm{AMI}=19,3 \pm 2,3 ; \mathrm{AR}=$ $18,6 \pm 3,2 ;$ ( $\mathrm{ng} / \mathrm{mg}$ tejido/30 $\mathrm{min}$ ); $\mathrm{p} \varangle 0,01 ; \mathrm{n}=10$ incubaciones para cada uno de los tres vasos estudiados y obtenidos de igual número de biopsias.

Pacientes diabéticos: En este grupo se observó un patrón de producción de prostaciclina distinto al obtenido en pacientes no diabéticos. En efecto, se observó que en condiciones basales la vena safena y la arteria mamaria intema sintetizaron cantidades signifi- cativamente menores de prostaciclina que la producida por estos dos vasos en pacientes no diabéticos. En cambio, la arteria radial de diabéticos produjo cantidades similares de prostaciclina a la sintetizada por esta arteria en no diabéticos, suginiendo que la capacidad biosintética de la arteria radial no se vio afectada por la presencia de esta enfermedad. Los resultados obtenidos se pueden observar en la Figura 1.

Suplementación con ácido araquidónico. Cuando se suplementó el medio de incubación con concentraciones de 10 a $50 \mu \mathrm{M}$ de este ácido se pudo observar que entre los vasos obtenidos de pacientes diabéticos, la que reaccionó mejor frente a la suplementación del medio fue la arteria radial. En efecto, $10 \mu \mathrm{M}$ de este ácido potenció la síntesis de prostaciclina en las

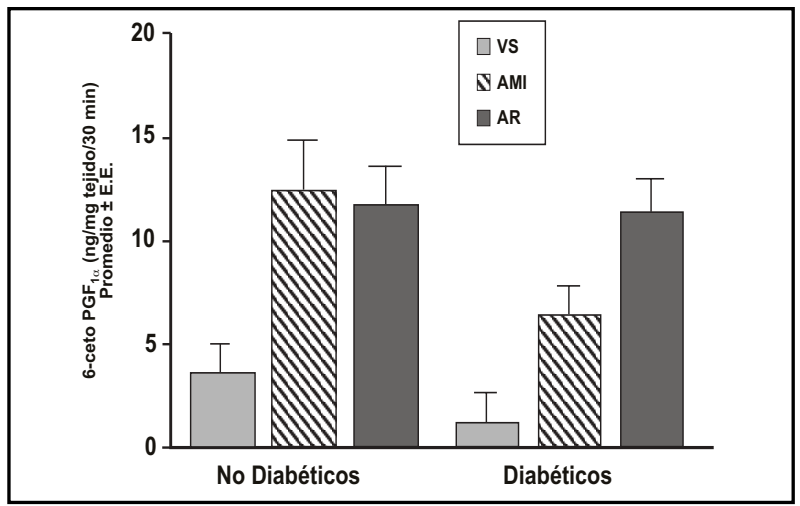

Figura 1. Comparación de la capacidad de la vena safena (VS) y arterias mamaria interna (AMI) y radial (AR) para sintetizar prostaciclina medida por radioinmunoensayo del 6-ceto-PGF1 $\alpha$ en pacientes diabéticos versus no diabéticos. Los valores corresponden al promedio \pm error estándar (E.E.) y se expresan en $\mathrm{ng} / \mathrm{mg}$ de tejido/30 min. $p<0,01$ para todas las comparaciones de los vasos entre no diabéticos y diabéticos; $n=10$ incubaciones para cada uno de los tres vasos estudiados y obtenidos de igual número de biopsias de cada grupo de pacientes. 


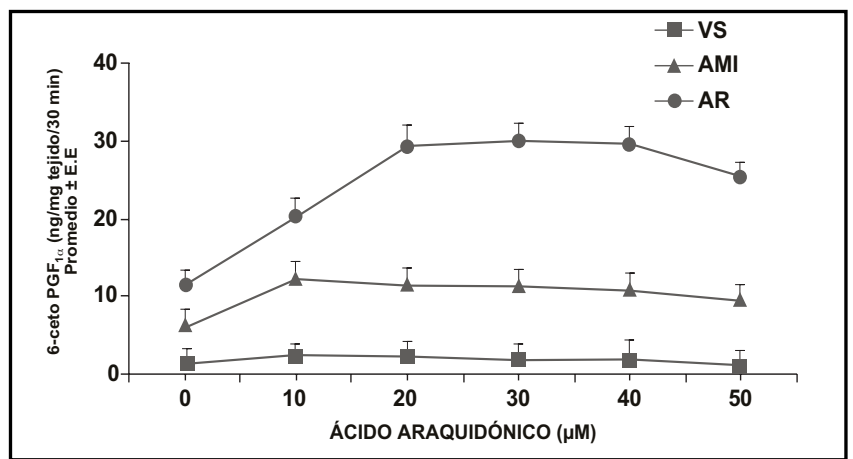

Figura 2. Conversión de diversas concentraciones de ácido araquidónico en prostaciclina en arterias mamaria interna y radial y vena safena obtenidas de diabéticos. Los valores corresponden al promedio \pm error estándar (E.E.) y se expresan en $\mathrm{ng} / \mathrm{mg}$ de tejido/30 min. $\mathrm{p}<0,01$ para todas las comparaciones; $\mathrm{n}=10$ incubaciones para cada uno de los tres vasos estudiados.

arterias mamaria interna y radial, no así en la vena safena; sin embargo, cuando se agregó al medio concentraciones mayores, se observó que sólo la arteria radial estimuló su producción de prostaciclina, no así la arteria mamaria interna y la vena safena que fueron incapaces de sintetizar mayores cantidades de prostaciclina (Figura 2). En el caso de estos dos últimos vasos, la máxima producción de prostaciclina se logró con $10 \mu \mathrm{M}$ de ácido araquidónico; en cambio, en el caso de la arteria radial se requinieron concentraciones entre 20 y $30 \mu \mathrm{M}$ para lograr la estimulación máxima. Consecuentemente, estos resultados evidencian que las concentraciones saturantes de este ácido fueron mayores en la arteria radial $(20-30 \mu \mathrm{M})$ que en la mamaria interna $(10 \mu \mathrm{M})$, suginiendo que la presencia de diabetes indujo una disminución de la capacidad sintética de producir prostaciclina en este último vaso, inhibiendo la actividad de la ciclooxigenasa u otras enzimas participantes en la síntesis de esta prostaglandina. No ocumió lo mismo con la arteria radial que probablemente debido a sus caractenísticas morfológicas y funcionales distintas a la mamania intema, conservó intacta esa capacidad.

\section{Discusión}

Nuestros resultados muestran que segmentos distales de la arteria mamaria interna y la vena safena de pacientes diabéticos sintetizan menores cantidades de prostaciclina que las de no diabéticos; en cambio, la arteria radial se comportó en forma distinta, pues produjo cantidades similares en ambos grupos. Esta acción se asoció con un aumento de la conversión del ácido araquidónico en prostaciclina cuando se suplementó el medio de incubación con distintas concentraciones de este ácido precursor de prostaciclina. En efecto, sus concentraciones saturantes fueron mayores en la arteria radial $(20 \mu \mathrm{M})$ que en la mamaria interna y vena safena $(10 \mu \mathrm{M})$, suginiendo que su capacidad biosintética no fue afectada por la presencia de diabetes.

La cinugía convencional de revasculanización utiliza una combinación de conductos venosos y arteriales, siendo los más usados la vena safena y la arteria mamaria intema ${ }^{19}$. Lamentablemente, la primera presenta una pobre permeabilidad a largo plazo, sufriendo reestenosis y aterosclerosis ${ }^{20}$. Aproximadamente $50 \%$ de los bypass con vena safena se ocluyen durante los primeros 10 años, en cambio con la arteria mamaria intema, ello ocume sólo en 5\%21. Estudios recientes muestran que la arteria radial constituye un excelente bypass coronario 22 , particularmente como conducto secundario o altemativo ${ }^{23}$, evidenciando buenos resultados clínicos ${ }^{24}$ que se asocian con una menor tasa de oclusión a los 6 meses $^{25}, 1$ año ${ }^{26}$ y 5 años de la cirugía coronaria ${ }^{27}$ y con elevados índices de sobrevivencia después del tercer año de revascularización ${ }^{2}$.

El endotelio vascular ejerce importantes funciones en la homeostasis circulatoria liberando sustancias vasodilatadoras, a saber, la prostaciclina y el óxido nítrico y vasoconstrictoras como el tromboxano y la endotelina. Estos productos endoteliales regulan el tono vascular, agregación plaquetaria, migración y proliferación de células musculares lisas y la adhesión de leucocitos al endotelio ${ }^{28}$, habiéndose planteado que la disfunción endotelial representa un factor clave en la permeabilidad y comporta- 
miento a largo plazo de los bypass comnarios. Los mecanismos responsables de estos últimos se han relacionado mayoritariamente con su capacidad de sintetizar prostaglandinas vasodilatadoras y antiagregantes plaquetarias, tales como la prostaciclina ${ }^{29}$. La relajación de las arterias coronarias a la hipoxia es dependiente de prostaglandinas ${ }^{30}$ y el balance entre prostaciclina y tromboxano es de gran importancia para lograr conductos vasculares que no se ocluyan a corto plazo ${ }^{10}$. Por otra parte, las arterias son capaces de sintetizar mayores cantidades de prostaciclina que las venas, tanto en las células endoteliales como en las musculares lisas ${ }^{3}$.

La diabetes induce disfunción endotelial ${ }^{31}$, factor clave en la tendencia a la reestenosis de los bypass coronarios. Los diabéticos muestran una alta incidencia de eventos postoperatorios desfavorables, incluyendo una disminución de la permeabilidad de los conductos implantados ${ }^{32}$. La vena safena de diabéticos sintetiza menos prostaciclina que la de no diabéticos debido a una deficiencia de la enzima ciclooxigenasa ${ }^{33}$, habiéndose observado un deterioro de su respuesta vasodilatadora y de la íntima vascular, incluyendo placas ateroscleróticas calcificadas $^{34}$. Por otra parte, la arteria mamaria interna muestra una menor relajación y producción de prostaciclina que la de no diabéticos, determinando cambios a lango plazo de estos conductos coronarios $^{16}$. Se ha postulado que alteraciones de las prostaglandinas sintetizadas por la ciclooxigenasa participan en la disfunción endotelial inducida por la diabetes. En efecto, la relajación a la hipoxia de la arteria radial es mayor que la de la mamaria interna y se acompaña de un aumento de prostaciclina; en cambio, la producción de tromboxano vasoconstrictor y agregante plaquetario es mayor en esta última que en la primera ${ }^{35}$. La resistencia a insulina reduce en el endotelio la actividad de la enzima prostaciclina sintetasa ${ }^{36}$ y la hiperglicemia aumenta la expresión de la ciclooxigenasa determinando un aumento del tromboxano ${ }^{14}$.

Nuestros resultados evidencian que en diabéticos, el comportamiento de la arteria radial y la mamaria interna es diferente, presentando esta última una reducción en la producción de prostaciclina, no así la radial que conservó su capacidad biosintética y produjo cantidades similares en diabéticos y no diabéticos. Consecuentemente, su relajación dependiente de prostaciclina es mayor que la de la mamaria interna y vena safena, suginiendo que la arteria radial representa un óptimo bypass complementario en diabéticos. Estas ventajas podrían estar relacionadas con algunas diferencias anatómicas entre ambas arterias ${ }^{37}$, con una mayor producción de prostaciclina ${ }^{5} 0$ con una sensibilidad mayor de la arteria radial a esta última. En cualquier caso, las propiedades favorables de los conductos radiales en diabéticos implicaría una menor tendencia a la aterosclemosis y oclusión a largo plazo.

Interesantemente, esta mayor capacidad de la arteria radial para sintetizar prostaciclina en diabéticos, se asoció con un aumento de la conversión de ácido araquidónico en esta prostaglandina. En efecto, nuestros resultados muestran que la suplementación del medio de incubación con distintas concentraciones de ácido araquidónico produjo efectos desiguales en estos vasos. La mayor producción de prostaciclina en la arteria radial se manifestó incluso después de suplementar el medio con ácido araquidónico en concentraciones saturantes de $20 \mu \mathrm{M}$; en el caso de la safena y mamaria interna, la máxima producción de prostaciclina se logró con concentraciones de $10 \mu \mathrm{M}$. Es decir, la arteria radial conserva en diabéticos su capacidad para generar prostaciclina y esta acción se ejerceńa en un sitio de la vía biosintética posterior a la liberación del ácido araquidónico desde los fosfolípidos de la membrana, probablemente a nivel de la enzima ciclooxigenasa. Esta evidencia no excluye la posibilidad de que esta arteria pueda liberar en forma más eficiente el ácido araquidónico, debido a la mayor cantidad de enzima fosfolipasa A2 de su tejido muscular. En otras palabras, la actividad intrínseca de las enzimas responsables de la síntesis de prostaciclina, a saber, fosfolipasa A2, ciclooxigenasa y prostaciclina sintetasa, seńa mayor en la arteria radial de diabéticos que en los otros vasos los cuales carecenín de la batería enzimática necesaria para transformar concentraciones elevadas de ácido araquidónico en prostaciclina. Estos resultados despiertan el interés por investigar la participación de otros productos endoteliales, particulamente el óxido nítrico, en la permeabilidad a largo plazo de los conductos coronarios utilizados en pacientes diabéticos. Este tópico, que no representó el objetivo principal de este trabajo, representa sin duda una línea de investigación relevante a dilucidar en estudios futuros.

Es importante destacar que la prostaciclina fue escogida como blanco de esta investigación en atención a que constituye el principal eicosanoide sintetizado por el endotelio vascular, representando, 
en conjunto con el óxido nítrico, los factores claves responsables del pronóstico a largo plazo que presentan los conductos utilizados como bypass coronarios. En efecto, la producción de prostaciclina asume gran relevancia en relación con el desamollo de la enfermedad aterosclerótica y tromboembólica debido a que constituye un potente inhibidor endógeno de la agregación plaquetaria, además de ser un intenso vasodilatador y modulador del metabolismo del colesterol. Por otra parte, la proliferación del músculo liso vascular juega un importante papel en el desarrollo de aterosclerosis; la prostaciclina es capaz de inhibir dicha proliferación ${ }^{8}$. Estas acciones que confirman el rol protector cardiovascular de la prostaciclina se manifiestan en mayor medida en aquellos vasos que tienen mayor capacidad para sintetizarla, tales como la arteria radial en el caso de sujetos diabéticos de acuerdo a nuestros resultados. En conjunto con la prostaciclina, otro derivado del ácido araquidónico, el tromboxano sintetizado por las plaquetas participa en el control de la reactividad vascular. Este último presenta acciones exactamente opuestas a la prostaciclina; en efecto, es un potente agregante plaquetario y vasoconstrictor, representando un agente de alto riesgo cardiovascular; el balance entre ambos eicosanoides es responsable en parte de la hemostasia vascular. Una producción vascular deficiente de prostaciclina expondrá localmente la superficie de los vasos a una acumulación de

\section{REFERENCIAS}

1. Lytle BW, Loop FD, Cosgrove DM, Ratuff NB, Easley $\mathrm{K}$, TAYLOR PC. Long-term serial studies of internal mammary artery and saphenous vein coronary bypass grafts. J Thorac Cardiovasc Surg 1985; 89: 248-58.

2. Zacharias A, Habib RH, Schwann TA, Riordan CJ, Durham SJ, SHAH A. Improved survival with radial artery versus vein conduits in coronary bypass surgery with left internal thoracic artery to left anterior descending artery grafting. Circulation 2004; 109: 1489-96.

3. Chakhouni A, Crawford FA, Kochel PJ, Olanoff LS, HalushKa PV. Human internal mammary artery produces more prostacyclin than saphenous vein. J Thorac Cardiovasc Surg 1986; 92: 88-91.

4. Berr ML, Guivernau M, Franck R, Piwonka G, ZamoraNO J, EDwARDs A. Producción comparativa de prostaciclina en arteria epigástrica inferior, arteria mamaria interna y vena safena: Implicancias en el pronóstico de la revascularización miocárdica. Rev Chil Cardiol 1992; 11: 13-9. plaquetas y a una producción exagerada de tromboxano y otros factores plaquetarios que estimularán la proliferación de células musculares lisas dentro de la pared, iniciando la secuencia de eventos que culminarán con la proliferación de la íntima, trombosis y aterosclerosis ${ }^{8}$. La posibilidad de manipular farmacológicamente el balance entre tromboxano y prostaciclina en favor de esta última sustancia representa, sin duda, un interesante mecanismo dirigido a optimizar el pronóstico de los conductos vasculares utilizados como bypass coronarios.

En conclusión, este trabajo muestra que la arteria radial es capaz de sintetizar mayores cantidades de prostaciclina que la vena safena y arteria mamaria interna en pacientes diabéticos y que este efecto se asocia con un aumento de la conversión del ácido araquidónico precursor en esta prostaglandina vasodilatadora y antiagregante plaquetaria. En atención a que estas dos últimas acciones representan factores claves en la permeabilidad a largo plazo de los bypass coronarios, surge la posibilidad que la arteria radial represente un excelente conducto complementario en diabéticos. Sus propiedades favorables y potencial menor tasa de oclusión asumirían particular relevancia en relación con un pronóstico más óptimo de la cirugía de revascularización miocárdica realizada en estos enfermos.

5. Guivernau M, Berr ML. Producción de prostaciclina en vasos usados en revascularización del miocardio. Rev Chil Cardiol 1999; 18: 143.

6. Manabe S, Sunamori M. Radial Artery Graft for Coronary Artery Bypass Surgery: Biological Characteristics and Clinical Outcome. J Card Surg 2006; 21: 102-14.

7. Weinschelbaum EE, Macchia A, Caramutti VM, Machain A, RAFAeLi HA, Favaloro MR et AL. Myocardial revascularization with radial and mammary arteries: initial and mid-term results. Ann Thorac Surg 2000; 70: 1378-83.

8. Moncada S, Vane JR. Pharmacology and endogenous roles of prostacyclin endoperoxides, thromboxane A2 and prostacyclin. Pharmacol Rev 1979; 30: 293-331.

9. LLRENS S, JoRDAN J, NAVA E. The nitric oxide pathway in the cardiovascular system. J Physiol Biochem 2002; 58: 179-88.

10. Chardigny CI, Van Der Perre K, Simonet S, Descombes J, FABIANi J, VerbeuRen TJ. Platelet and prostacyclin in arterial bypasses: implications for coronary artery surgery. Ann Thorac Surg 2000; 69: 513-9.

11. Cheng Y, Austin S, Rocca B, Koler B, Coffman T, 
Grosser T, Lawson J. Role of prostacyclin in the cardiovascular response to thromboxane A2. Science 2002; 296: 539-41.

12. Connoluy E, Bouchier-Hayes DJ, Kaye E, Leahy A, Fitzgerald D, Belton O. Cyclooxygenase Isozyme Expression and Intimal Hyperplasia in a Rat Model of Balloon Angioplasty. J Pharmacol Exp Ther 2002; 300: 393-8.

13. Timms AD. Diabetes: Relationship to ischaemic heart disease. British Medical Bulletin 2001; 59: 159-72.

14. Cosentino F, Eto M, De Paolis P, van der Loo B, BACHSCHMID M, UluRICH V ET AL. High glucose causes upregulation of cyclooxygenase-2 and alters prostanoid profile in human endothelial cells. Circulation 2003; 107: 1017-23.

15. Santimi F, Davi G, Consoli A, Cipolone F, Mezzetti A, FALCO A ET AL. Thromboxane-Dependent CD40 Ligand Release in Type 2 Diabetes Mellitus. J Am Coll Cardiol 2006; 47: 391-7.

16. Pompilio G, Rossoni G, Alamanni F, Tartara P, Barajon I, Rumio C et aL. Comparison of endotheliumdependent vasoactivity of internal mammary arteries from hypertensive, hypercholesterolemic and diabetic patients. Ann Thorac Surg 2001; 72: 1290-7.

17. Chardigny C, Jebera VA, Acar C, Descombes JJ, VerBEUREN TJ, CARPENTIER A, FABIANI JN. Vasoreactivity of the radial artery. Comparison with the internal mammary and gastroepiploic arteries with implications for coronary artery surgery. Circulation 1993; 88: II115-II127.

18. Guivernau M, Armijo F, Rosas R. Role of sulfhydryl groups in the stimulatory effect of captopril on vascular prostacyclin synthesis. Eur J Pharmacol 1991; 198: 1-6.

19. Van Der Meer J, Hiliege HL, Van Gilst WH, Brutel De LA Riviere A, Dunselman PH, Fidier V et AL. A comparison of internal mammary artery and saphenous vein grafts after coronary artery bypass surgery. Circulation 1994; 90: 2367-74.

20. Motwani JG, Topol EJ. Aortocoronary saphenous vein graft disease: Pathogenesis, predisposition and prevention. Circulation 1998; 97: 916-31.

21. Grondin CM, Campeau L, Thornton JC, Engle JC, Cross FS, SCHREIBER H. Coronary artery bypass grafting with saphenous vein. Circulation 1989; 79: 124-9.

22. SajJa LR, Mannam G, Pantula NR, Sompadi S. Role of radial artery graft in coronary artery bypass grafting. Ann Thorac Surg 2005; 79: 2180-8.

23. Mussa S, TAGgaRT DP. Radial artery conduits for coronary artery bypass grafting: Current perspective. J Thorac Cardiovasc Surg 2005; 129: 250-3.

24. Nezic DG, Knezevic AM, Milojevic PS, Tukanovic BP, Jovic M, Borzanovic M, Nezkovic AN. The fate of the radial artery conduit in coronary artery bypass grafting surgery. Eur J Cardiothorac Surg 2006; 30: 341-6.

25. Al-Bustami MH, Amrani M, Chester AH, Lisley CJ, YACOUB MH. In vivo early and midterm flow-mediated endothelial function of the radial artery used as a coronary bypass graft. J Am Coll Cardiol 2002; 39: 573-7.

26. Desai ND, Cohen EA, Naylor CD, Fremes SE. A randomized comparison of radial artery and saphenous vein coronary bypass grafts. N Engl J Med 2004; 351: 2302-9.

27. Cameron J, Trivedi S, Stafford G, Bett N. Five-Year Angiographic Patency of Radial Artery Bypass Grafts. Circulation 2004; 110: II-23-II-26.

28. Gimbrone MA, TOPPER JN. Biology of the vessel wall: endothelium. En: K.R. Chien, Editor, Molecular basis of heart disease, Hardcourt Brace \& Co, Troy, MO 1998, pp. 331-48.

29. Rudic D, Brinster D, Cheng Y, Fries S, Song W, Austin $\mathrm{S}$ ET AL. COX-2-derived prostacyclin modulates vascular remodeling. Circulation Research 2005; 96: 1240-7.

30. JiANG C, COWNS P. Inhibition of hypoxia-induced relaxation of rabbit isolated coronary arteries. $\mathrm{Br} \mathrm{J}$ Pharmacol 1994; 111: 711-6.

31. Calues-Escandon J, Cipola M. Diabetes and endothelial dysfunction: a clinical perspective. Endocr Rev 2001; 22: 36-52.

32. MAK KH, FAXON DP. Clinical studies on coronary revascularization in patients with type 2 diabetes. Eur Heart J 2003; 24: 1087-103.

33. Brunkwall JS, Berggvist D. Prostacyclin release from the human saphenous vein in diabetics is lower than in nondiabetics. World J Surg 1992; 16: 1141-5.

34. Lorusso R, Pentiricci S, Raddino R, Scarabelu TM, ZambeLI C, ViLANACCI V ET aL. Influence of type 2 diabetes on functional and structural properties of coronary artery bypass conduits. Diabetes 2003; 52: 2814-20.

35. Gupte SA, Zias EA, Sarabu MR, Wolin MS. Role of prostaglandins in mediating differences in human internal mammary and radial artery relaxation elicited by hypoxia. J Pharmacol Exp Ther 2004; 311: 510-8.

36. Du X, Edelstein D, Obici S, Higham N, Zou M, BROWNLEE M. Insulin resistance reduces arterial prostacyclin synthase and NOS activities by increasing endothelial fatty acid oxidation. J Clin Invest 2006; 116: 1071-80.

37. Van Son JA, Smedts F, Vincent JG, Van Lier HJ, Kubat $\mathrm{K}$. Comparative anatomical studies of various arterial conduits for myocardial revascularization. J Thorac Cardiovasc Surg 1990; 99: 703-7. 